\title{
Aliskiren attenuates cardiac dysfunction by modulation of the mTOR and apoptosis pathways
}

\author{
Zhengbo Zhao (ii) ${ }^{1 *}$, Han Liu (iD) ${ }^{2 *}$, and Dongmei Guo (i) ${ }^{3}$ \\ ${ }^{1}$ Department of Cardiovascular Medicine, Jiulongpo District People's Hospital, Chongqing, China \\ ${ }^{2}$ Department of Neurology, Jiulongpo District People's Hospital, Chongqing, China \\ ${ }^{3}$ Department of Cardiovascular Medicine, Nanchuan District People's Hospital, Chongqing, China
}

\begin{abstract}
Aliskiren (ALS) is well known for its antihypertensive properties. However, the potential underlying the molecular mechanism and the anti-hypertrophic effect of ALS have not yet been fully elucidated. The aim of the present study was to investigate the role of ALS in mammalian target of rapamycin (mTOR) and apoptosis signaling using in vivo and in vitro models of cardiac hypertrophy. A rat model of cardiac hypertrophy was induced by isoproterenol treatment $\left(5 \mathrm{mg} \cdot \mathrm{kg}^{-1} \cdot \mathrm{day}^{-1}\right)$ for 4 weeks, with or without ALS treatment at $20 \mathrm{mg} \cdot \mathrm{kg}^{-1} \cdot \mathrm{day}^{-1}$. The expression of hypertrophic, fibrotic, and apoptotic markers was determined by RT-qPCR. The protein expression of apoptotic markers mTOR and p-mTOR was assessed by western blot analysis. The proliferation of $\mathrm{H} 9 \mathrm{C} 2$ cells was monitored using the MTS assay. Cell apoptosis was analyzed using flow cytometry. In vivo, isoproterenol-treated rats exhibited worse cardiac function, whereas ALS treatment reversed these dysfunctions, which were associated with changes in p-mTOR, Bcl-2, Bax, and cleaved caspase-3 expression, as well as the number of apoptotic cells. In vitro, H9C2 cardiomyocyte viability was significantly inhibited and cardiac hypertrophy was induced by Ang II administration, but ALS reversed Ang II-induced H9C2 cardiomyocyte hypertrophy and death. Furthermore, Ang II triggered the activation of the mTOR and apoptosis pathways in hypertrophic cardiomyocytes that were inhibited by ALS treatment. These results indicated that ALS alleviated cardiac hypertrophy through inhibition of the mTOR and apoptosis pathways in cardiomyocytes.
\end{abstract}

Key words: Aliskiren; Cardiac hypertrophy; Fibrosis; Mammalian target of rapamycin; Apoptosis

\section{Introduction}

Cardiac hypertrophy is one of the main causes of cardiovascular morbidity and mortality, and its prevention may represent a new management strategy for improving survival in patients with cardiovascular diseases (CVDs) (1). The pathological mechanisms of cardiac hypertrophy involve various molecular and signaling pathways, including those associated with oxidative stress (2), autophagy (3), and inflammatory response (4). The mammalian target of rapamycin (mTOR) signaling cascade is implicated in the induction of cardiac hypertrophy and fibrosis (5). Several in vivo and in vitro studies have documented that the mTOR pathway is a promising therapeutic target for pressure overload-, isoproterenol (ISO)- and angiotensin (Ang) Il-induced cardiac hypertrophy, and fibrosis (6-8). However, Song et al. demonstrated that mTOR attenuates pressure overload-induced cardiac dysfunction and hypertrophy by regulation of the inflammatory reaction (9). Kemi et al. (10) demonstrated that pressure overload is associated with inactivation of the mTOR pathway. These findings suggest that the role of mTOR in the pathological process of cardiac hypertrophy and fibrosis has not been fully defined.

The renin-angiotensin system (RAS) and Ang II, a key active peptide in RAS, have been shown to be closely associated with cardiac hypertrophy and fibrosis, which may be prevented by angiotensin-converting enzyme inhibitor (ACEI) or Ang II type 1 receptor blocker (ARB) treatment $(11,12)$. However, ACEI and ARB only partially protect against the progression of CVDs, which may be attributed to the increase in plasma renin activity and the production of Ang I (13). This phenomenon may lead to 'ACE escape' and restore the circulating Ang II to baseline levels in patients receiving long-term treatment with ACEI or ARB (14). Aliskiren (ALS) is a renin inhibitor that prevents the formation of Ang I from angiotensinogen, and may represent a novel approach to restricting RAS by

Correspondence: Dongmei Guo: <guodongmei677@163.com>

${ }^{*}$ These authors contributed equally to this study.

Received June 16, 2019 | Accepted October 21, 2019 
directly inhibiting this system at its rate-limiting proximal step (15). Previous studies reported that ALS is as effective as ACEI or ARB in controlling blood pressure $(1,16,17)$. Recently, the antihypertrophic and antifibrotic effects of ALS have been emerging in experimental and clinical studies $(1,14,18)$. However, the mechanisms underlying the therapeutic effects of ALS on cardiac hypertrophy and fibrosis remain poorly understood.

The aim of the present study was to investigate whether ALS protects against ISO-induced cardiac hypertrophy and fibrosis in a rat model and Ang II-induced cardiac hypertrophy in vitro. The apoptotic mechanism and AKT/mTOR pathway, which may contribute to the antihypertrophic and antifibrotic effects of ALS in vivo and in vitro, were also investigated.

\section{Material and Methods}

\section{Animal treatment}

A total of 24 ten-week-old male Sprague-Dawley rats (body weight 200-250 g) were purchased from Vital River Laboratories Co., Ltd. (China) and allowed to acclimatize to the environment for 1 week. The rats were randomly divided into four groups: Vehicle group rats $(n=6)$ received normal saline by subcutaneous injection; ISO group rats $(n=6)$ were treated with ISO (Santa Cruz Biotechnology, USA) at a dose of $5 \mathrm{mg} / \mathrm{kg}$ per day by subcutaneous injection for 2 weeks to induce cardiac hypertrophy as described previously $(19,20)$; ALS group rats $(n=6)$ were treated with ALS (cat. No. CDS023114; purity: $\geqslant 98 \%$; Sigma-Aldrich; Merck KGaA, USA; formula: $\mathrm{C} 30 \mathrm{H} 53 \mathrm{~N}$ 306) at a dose of $20 \mathrm{mg} / \mathrm{kg}$ per day by intragastric administration; and ALS + ISO group rats $(n=6)$ were treated with ISO (5 mg/kg per day) combined with ALS $\left(20 \mathrm{mg} \cdot \mathrm{kg}^{-1} \cdot \mathrm{day}^{-1}\right)$. All the rats were sacrificed by an overdose of sodium pentobarbital $(2 \% ; 200 \mathrm{mg} / \mathrm{kg}$; SigmaAldrich; Merck KGaA) after 4 weeks of treatment. The hearts were collected and immediately weighed, and then frozen in liquid nitrogen for gene and protein analysis, or fixed in $4 \%$ formalin at room temperature and embedded in paraffin for histological analysis. The experiments were approved by the Ethics Committee of the Nanchuan District People's Hospital (China).

\section{Hematoxylin and eosin (H\&E) staining}

The left ventricles (LVs) were collected and fixed with $4 \%$ formalin at room temperature for $24 \mathrm{~h}$, and then embedded in paraffin. Tissues were cut into $3-\mu \mathrm{m}$ sections, which were stained with H\&E (Beyotime Institute of Biotechnology, China) at room temperature and visualized under a microscope (Leica DM 2500; Leica Microsystems $\mathrm{GmbH}$, Germany). The width of cardiomyocytes was measured with an automated image analysis system (Image-Pro Plus 5.0, Media Cybernetics, USA).

\section{RNA extraction and reverse transcription-quantitative polymerase chain reaction (RT-qPCR) analysis}

Total RNA was extracted using TRIzol ${ }^{\circledR}$ (Invitrogen; Thermo Fisher Scientific, Inc., USA), according to the manufacturer's protocol. cDNA was synthesized by reverse transcription reactions with $2 \mu \mathrm{g}$ total RNA using Moloney Murine Leukemia Virus Reverse Transcriptase (Invitrogen; Thermo Fisher Scientific, Inc.). PCR analysis was performed using the TaqMan Universal PCR Master Mix (Thermo Fisher Scientific, Inc.) with a DNA Engine (ABI 7300; Thermo Fisher Scientific, Inc.). The reaction conditions were as recommended by the manufacturer's protocol as follows: $95^{\circ} \mathrm{C}$ for $10 \mathrm{~min}, 35$ cycles of $95^{\circ} \mathrm{C}$ for $15 \mathrm{~s}, 60^{\circ} \mathrm{C}$ for $30 \mathrm{~s}$, and $72^{\circ} \mathrm{C}$ for $30 \mathrm{~s}$. The PCR primers are shown in Table 1. Glyceraldehyde-3-phosphate dehydrogenase (GAPDH) levels were used to normalize the expression of the target genes. The relative gene expression levels were calculated using the $2^{-\Delta \Delta \mathrm{Cq}}$ method (21).

\section{Western blotting}

Protein was extracted using RIPA Lysis buffer (Beyotime Institute of Biotechnology, China). The concentration was determined using the Bicinchoninic Acid Kit for Protein

Table 1. Primers used in the RT-qPCR.

\begin{tabular}{lcc}
\hline Gene & Forward primer $\left(5^{\prime}-3^{\prime}\right)$ & Reverse primer $\left(5^{\prime}-3^{\prime}\right)$ \\
\hline ANP & GGAGCCTGCGAAGGTCAA & TATCTTCGGTACCGGAAGCTGT \\
BNP & CAGAAGCTGCTGGAGCTGATAAG & TGTAGGGCCTTGGTCCTTTG \\
$\alpha-M H C$ & GCCCTTTGACATCCGCACAGAGT & TCTGCTGCATCACCTGGTCCTCC \\
$\beta-M H C$ & GCGGACATTGCCGAGTCCCAG & GCTCCAGGTCTCAGGGCTTCACA \\
Procollagen I & TATGCTTGATCTGTATCTGCCACAAT & TCGCCCTCCCGTTTTTG \\
Procollagen III & CAGCTGGCCTTCCTCAGACT & TGCTGTTTTTGCAGTGGTATGTAA \\
Bcl-2 & GAGCGTCAACAGGGAGATGT & CAGCCAGGAGAAATCAAACAG \\
Bax & TTGCTACAGGGTTTCATCCA & TGTTGTTGTCCAGTTCATCG \\
Caspase3 & AGCTGGACTGCGGTATTGAG & AGCTGGACTGCGGTATTGAG \\
GAPDH & GCACCGTCAAGCTGAGAAC & TGGTGAAGACGCCAGTGGA \\
\hline
\end{tabular}


Determination (Sigma-Aldrich; Merck KGaA). Samples containing $30 \mu \mathrm{g}$ protein were separated by $10 \%$ SDSPAGE and transferred onto nitrocellulose membranes (BioRad Laboratories, Inc., USA). Primary antibodies against mTOR (cat. No. sc-293089, dilution, 1:1000), p-mTOR (cat. No. sc-293132, dilution, 1:500), Bcl-2 (cat. No. sc56015, dilution, 1:1000) and Bax (cat. No. sc-6236, dilution, 1:1000) were purchased from Santa Cruz
Biotechnology. Cleaved caspase-3 (cat. No. 9661, dilution, 1:1000) was purchased from Cell Signaling Technology, Inc. (USA). After incubation with primary antibodies at room temperature for $2 \mathrm{~h}$, the membranes were incubated with the appropriate horseradish peroxidaseconjugated secondary antibody (cat. No. sc-516102; dilution, 1:10,000; Santa Cruz Biotechnology), following visualization using chemiluminescence (Thermo Fisher

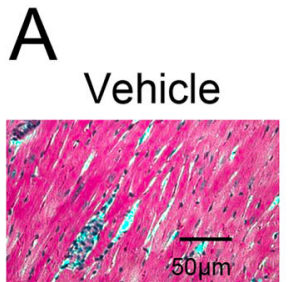

ALS

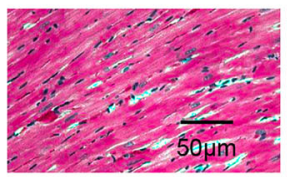

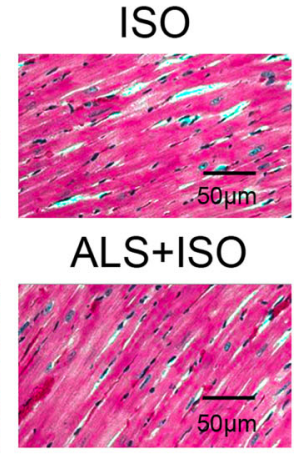
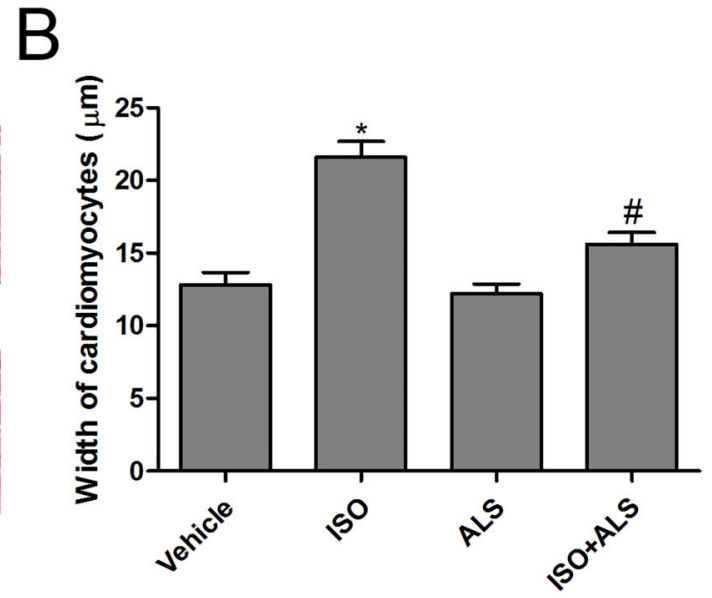

Figure 1. Effect of aliskiren (ALS) on cardiomyocyte size in isoproterenol (ISO)-treated rats. A, Hematoxylin and eosin staining was performed and (B) the width of cardiomyocytes was measured ( $n=6$ per group). Data are reported as means $\pm S E$. ${ }^{*} P<0.05$ compared with vehicle; ${ }^{\#} \mathrm{P}<0.05$ compared with the ISO-treated group (ANOVA followed by Tukey's post hoc test).

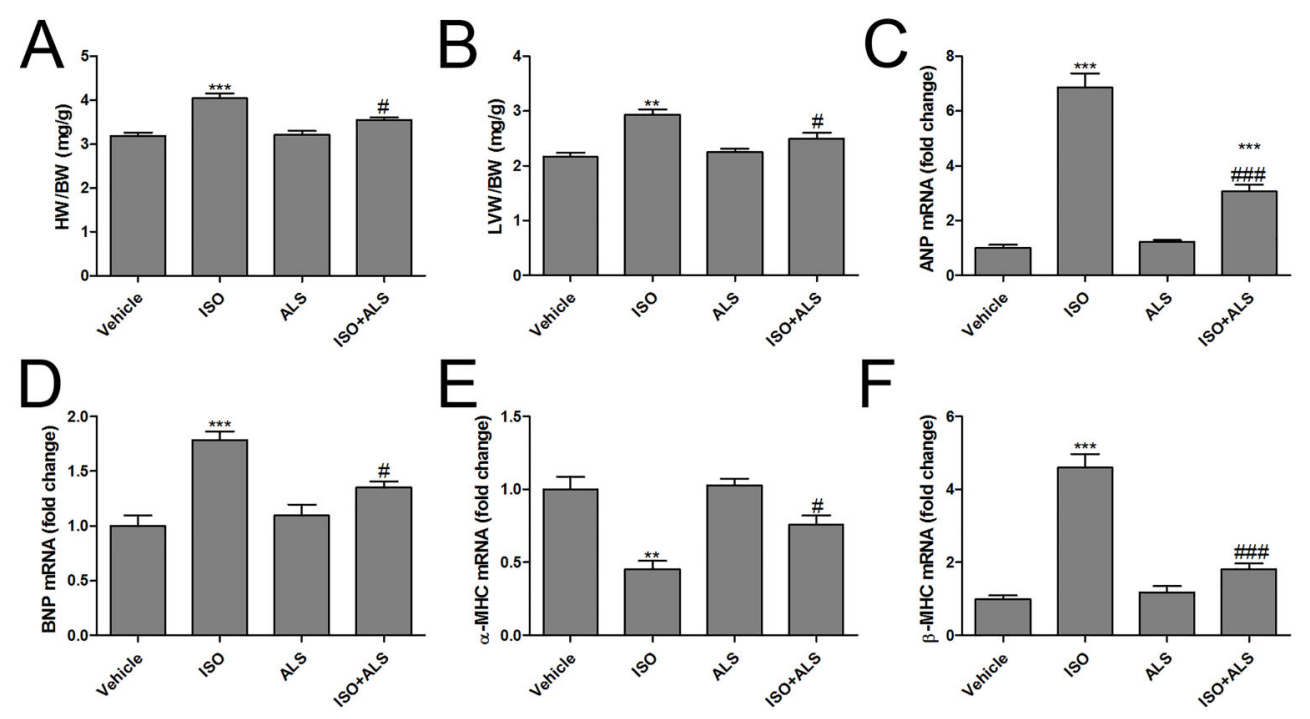

Figure 2. Canonical markers of cardiac hypertrophy in response to aliskiren (ALS) in isoproterenol (ISO)-treated rats. The ratio of (A) heart weight/body weight (HW/BW) and (B) left ventricular weight to body weight (LVW/BW), the mRNA levels of (C) atrial natriuretic peptide (ANP), (D) brain natriuretic peptide (BNP), (E) $\alpha$-myosin heavy chain $(\alpha-M H C)$, and $(\mathbf{F}) \beta-M H C$ were measured in the left ventricles of the rats ( $n=6$ per group). Data are reported as means $\pm S E$. ${ }^{\star \star} P<0.01,{ }^{\star \star *} P<0.001$ compared with vehicle; ${ }^{*} P<0.05$, \#\# $\mathrm{P}<0.001$ compared with the ISO group (ANOVA followed by Tukey's post hoc test). 
Scientific, Inc.). $\beta$-actin (cat. No. sc-130301; dilution, 1:2000; Santa Cruz Biotechnology) was used as the control antibody. Signals were analyzed with Quantity One ${ }^{\circledR}$ software, version 4.5 (Bio-Rad Laboratories, Inc.).

\section{Cell culture}

H9C2 cells were purchased from the American Type Culture Collection (Bethesda, USA) and cultured in Dulbecco's modified Eagle's medium (DMEM; Gibco; Thermo Fisher Scientific, Inc., USA) containing 10\% fetal calf serum (Gibco; Thermo Fisher Scientific, Inc.), 10\% L-glutamine, $\quad 0.5 \%$ penicillin/streptomycin, $10 \%$ nonessential amino acids, and $10 \%$ pyruvate in a $5 \% \mathrm{CO}_{2}$ atmosphere at $37^{\circ} \mathrm{C}$. $\mathrm{H} 9 \mathrm{C} 2$ cells were treated with Ang II alone (10 $\mu \mathrm{M}$, Sigma-Aldrich; Merck KGaA) or combined with ALS $(20 \mu \mathrm{M})$ or rapamycin (RAP; $100 \mathrm{nM}$, SigmaAldrich; Merck KGaA). All the experiments were performed in triplicate.

\section{MTS assay}

The proliferation of $\mathrm{H} 9 \mathrm{C} 2$ cells was monitored using the MTS assay kit (Promega Corporation, USA). Absorbance was measured at $492 \mathrm{~nm}$ using an ELISA reader (MD SpectraMax M5; Molecular Devices, LLC, USA). The MTS assay was performed as described previously (22).

\section{Flow cytometry analysis}

H9C2 cells were treated with Ang II $(10 \mu \mathrm{M})$, Ang II $(10 \mu \mathrm{M})+$ ALS $(20 \mu \mathrm{M})$, or Ang II $(10 \mu \mathrm{M})+\operatorname{RAP}(100 \mathrm{nM})$ for $24 \mathrm{~h}$. Cells were collected after digestion, washed twice with PBS, and centrifuged at $1,500 \mathrm{~g}$ for $5 \mathrm{~min}$ at $4^{\circ} \mathrm{C}$. The supernatant was discarded, and the cells were resuspended, fixed in ice-cold $75 \%$ ethanol, and stored at $4^{\circ} \mathrm{C}$. The cell apoptosis assay was conducted as previously described (23). The Annexin V-FITC apoptosis detection kit was purchased from Invitrogen (Thermo Fisher Scientific). The samples were analyzed using a flow cytometer (BD Biosciences, USA). The data were processed by Cell Quest Software (version 5.1, BD Biosciences).

\section{Statistical analysis}

Data are reported as means \pm SE. Statistical analysis was performed using GraphPad Prism version 7.0 (GraphPad Software, Inc., USA). Inter-group differences were analyzed by one-way analysis of variance, followed by Tukey's post hoc analysis. $\mathrm{P}<0.05$ was considered to be a statistically significant difference.

\section{Results}

ALS attenuated ISO-induced cardiac hypertrophy and fibrosis in rats

To investigate the cardioprotective effects of ALS on ISO-induced cardiac hypertrophy and fibrosis in vivo, a rat model of cardiac hypertrophy and fibrosis was successfully constructed. First, it was observed that ISO induced marked cardiac hypertrophy by increasing the cardiomyocyte width, which was significantly suppressed in the ALS + ISO treatment group (Figure 1A and $\mathrm{B}$ ). In addition, both the ratio of heart weight to body weight (HW/BW) and LV weight to BW (LVW/BW) were increased following ISO injection, but HW/BW and LVW/BW were lowered in the combined ALS + ISO group (Figure 2A and $B$ ). Furthermore, the hypertrophic markers, including atrial natriuretic peptide (ANP) and brain natriuretic peptide (BNP), $\alpha$-myosin heavy chain $(\alpha-M H C)$ and $\beta$-myosin heavy chain $(\beta-M H C)$, were evaluated in the LVs of rats. RT-qPCR analysis demonstrated that the levels of ANP, BNP, and $\beta-M H C$ were significantly higher in the LVs from ISO-treated rats compared with the control group, but treatment with ALS significantly decreased the ISO-mediated increase in ANP, BNP, and $\beta-\mathrm{MHC}$ mRNA expression (Figure $2 \mathrm{C}$, $D$ and $F$ ). It was also observed that ISO treatment

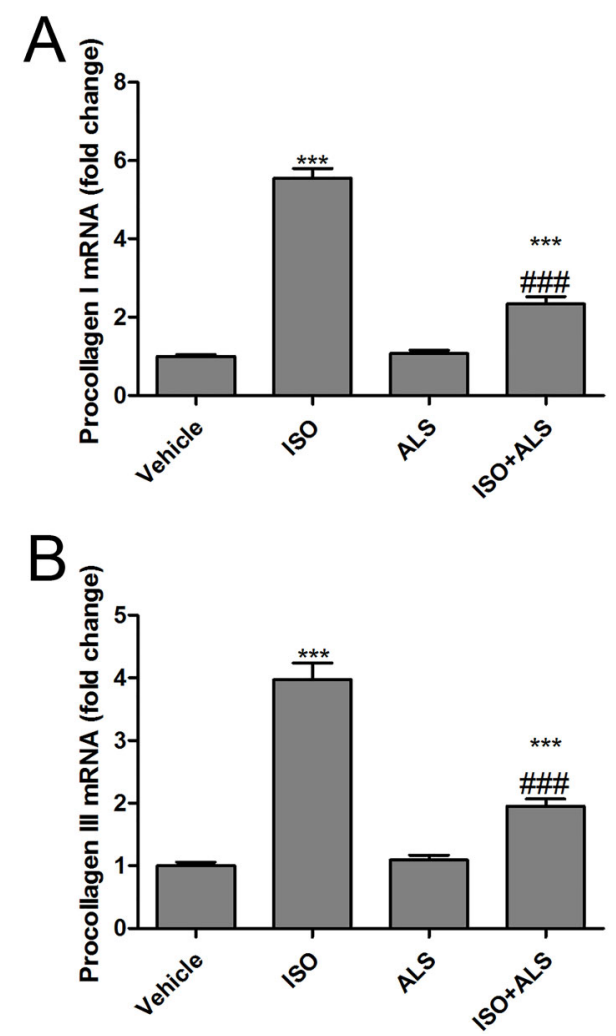

Figure 3. Canonical markers of cardiac fibrosis in response to aliskiren (ALS) in isoproterenol (ISO)-treated rats. The mRNA expression of (A) procollagen I and (B) procollagen III was measured by RT-qPCR in the left ventricles of the rats $(n=6$ per group). Data are reported as means $\pm S E$. ${ }^{* *} \mathrm{P}<0.001$ compared with vehicle; ${ }^{\prime \prime \prime} \mathrm{P}<0.001$ compared with the ISO group (ANOVA followed by Tukey's post hoc test). 
markedly decreased $\alpha$-MHC mRNA expression. However, ALS treatment significantly reversed the ISOinduced downregulation of $\alpha-\mathrm{MHC}$ in the LVs of rats (Figure 2E).

\section{Effect of ALS on fibrotic markers associated with cardiac hypertrophy}

To investigate the effect of ALS on fibrosis in ISOtreated rats, the cardiac gene expression of the fibrotic markers procollagen I and III was measured. The results demonstrated that ISO treatment significantly upregulated procollagen I and III mRNA expression compared with the control group, whereas ALS suppressed the fibrotic responses in LVs triggered by ISO (Figure $3 \mathrm{~A}$ and $B)$.

\section{Inhibition of apoptosis and mTOR signaling by ALS in} ISO-treated rats

To determine the molecular mechanisms of ALS in ISO-induced cardiac hypertrophy and fibrosis, the apoptosis and mTOR pathways were assessed by RT-qPCR and western blotting in isolated rat LVs. The apoptosis-related markers Bax and caspase-3, and the anti-apoptosis protein $\mathrm{Bcl}-2$ were evaluated. ISO increased the mRNA and protein expression of Bax and caspase-3, and suppressed the mRNA and protein expression of $\mathrm{Bcl}-2$ in isolated LVs; these effects were blocked by ALS treatment (Figure 4A, B, and C). Moreover, the phosphorylation levels of the $\mathrm{mTOR}$ protein exhibited a significant increase in the ISO-treated group. However, ALS treatment effectively inhibited the activation of the mTOR signaling pathway triggered by ISO (Figure 4D).

\section{ALS prevented Ang II-modulated apoptosis and mTOR signaling}

First, the hypertrophy markers ANP and BNP were investigated in Ang II-treated $\mathrm{H} 9 \mathrm{C} 2$ cardiomyocytes. The results suggested that Ang II treatment significantly upregulated the mRNA levels of ANP and BNP, suggesting that Ang II successfully induced hypertrophy in cardiomyocytes in vitro (Figure $5 \mathrm{~A}$ and $\mathrm{B}$ ). Next, the potential cytotoxicity of Ang II, ALS, and RAP was analyzed using an MTS assay. H9C2 cardiomyocytes were treated with Ang II, Ang II + ALS, or Ang II+RAP

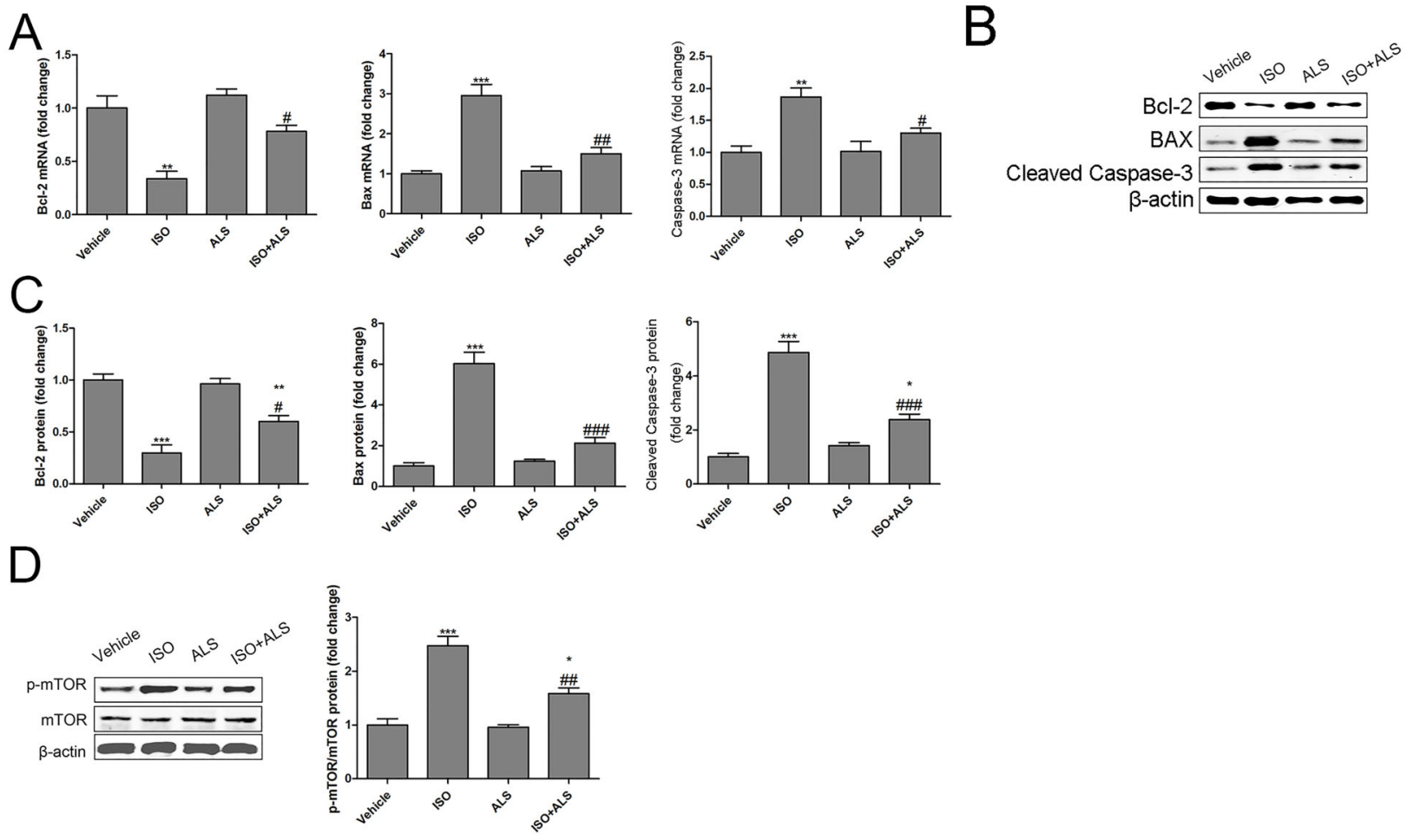

Figure 4. Aliskiren (ALS) inhibited the mTOR and apoptosis pathways in isoproterenol (ISO)-treated rats. The mRNA expression of $\mathrm{Bcl}-2$, Bax, and caspase-3 was measured by RT-qPCR in the left ventricles of the rats ( $\mathrm{n}=6$ per group) (A). The protein expression of $\mathrm{Bcl}-2$, Bax, and cleaved caspase-3 was measured by western blotting in the left ventricles of the rats (B and $\mathbf{C})$. The protein expression of mTOR and $p-m T O R$ was measured by western blotting in the left ventricles of the rats $(\mathbf{D})$ ( $n=6$ per group). Data are reported as means $\pm S E$. ${ }^{*} P<0.05$, ${ }^{* *} P<0.01$, ${ }^{* * *} P<0.001$ compared with vehicle; ${ }^{\#} P<0.05,{ }^{\# \#} P<0.01,{ }^{\# \#} P<0.001$ compared with the ISO group (ANOVA followed by Tukey's post hoc test). mTOR: mammalian target of rapamycin. 

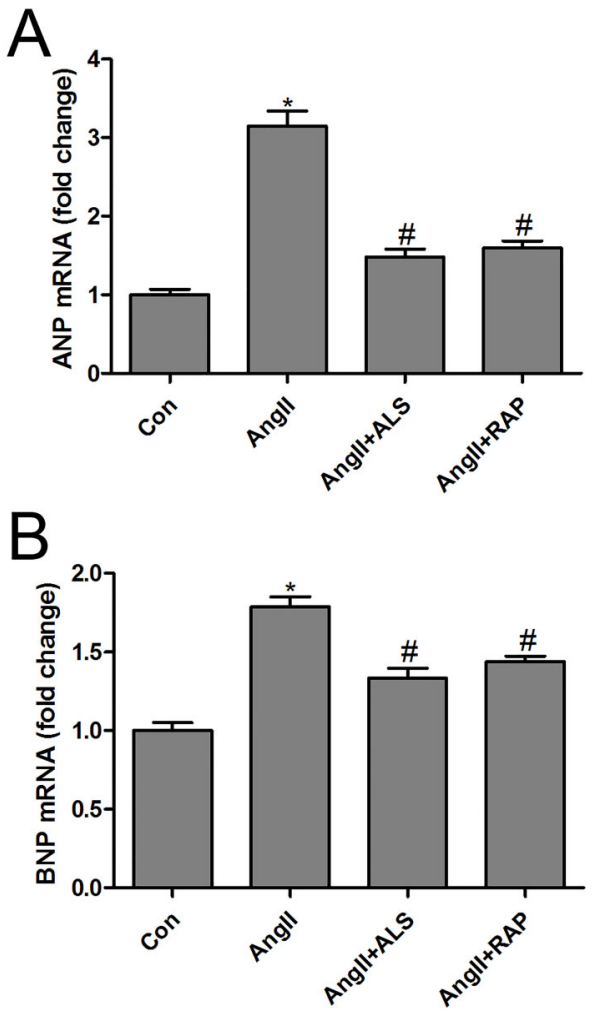

Figure 5. Aliskiren (ALS) decreased the markers of cardiac hypertrophy in Ang II-treated $\mathrm{H} 9 \mathrm{C} 2$ cardiomyocytes. The mRNA expression of (A) ANP and (B) BNP was measured by RT-qPCR in the $\mathrm{H} 9 \mathrm{C} 2$ cardiomyocytes after treatment with Ang II $(10 \mu \mathrm{M})$, Ang II $(10 \mu \mathrm{M})+$ ALS $(20 \mu \mathrm{M})$, or Ang II $(10 \mu \mathrm{M})+\operatorname{RAP}(100 \mathrm{nM})$ for $48 \mathrm{~h}(\mathrm{n}=3$ per group). Data are reported as means $\pm \mathrm{SE}$. ${ }^{*} \mathrm{P}<0.05$ compared with control; ${ }^{*} \mathrm{P}<0.05$ compared with the Ang II-treated group (ANOVA followed by Tukey's post hoc test). Ang: angiotensin; ANP: atrial natriuretic peptide; BNP: brain natriuretic peptide; RT-qPCR: reverse transcription-quantitative polymerase chain reaction.

for different times. Cell viability did not differ notably at $24 \mathrm{~h}$ of incubation. However, cell viability was significantly inhibited by Ang II at 48 and 72 h. Both ALS and RAP inhibited Ang II-induced H9C2 cardiomyocyte death (Figure 6A). Similar results were obtained by flow cytometry analysis after treatment with different conditions at $48 \mathrm{~h}$ (Figure 6B and $\mathrm{C}$ ). To confirm the modulatory effect of the apoptosis and mTOR pathways on the ALS-mediated cardioprotective effect against Ang II-induced cardiac hypertrophy in vitro, $\mathrm{H} 9 \mathrm{C} 2$ cardiomyocytes were treated with Ang II, ALS, or RAP (an inhibitor of mTOR). The results indicated that both ALS and RAP inhibited Ang Il-activated apoptosis and mTOR signaling in vitro (Figure 7A-D), which were consistent with the results in vivo. These data provided evidence that ALS treatment improved cardiac hypertrophy and fibrosis by inhibiting the mTOR and apoptosis pathways in vivo and in vitro.

\section{Discussion}

The findings of this study demonstrated that ALS attenuated ISO- and Ang II-induced cardiac dysfunction by inhibiting mTOR and apoptosis pathways. Previous studies showed that the effective dose of ALS ranges from 10 to $100 \mathrm{mg} / \mathrm{kg}$ per day and has a beneficial effect on cardiovascular diseases, including cardiac hypertrophy, in a rat model $(14,24)$.

In Ang II-induced H9C2 cardiomyocyte hypertrophy, the fold change of BNP mRNA ( 13-fold) was markedly higher compared with that of ANP ( $\sim 10$-fold) (25). Moreover, the fold change of ANP and BNP was $\sim 2.0$ and 1.2, respectively, after exposure to ISO; these results are comparable to the control group (26). More importantly, ISO induced upregulation of ANP, but not of BNP, in the LVs of the rats (27). In the present study, the change in ANP and BNP was 3.1- and 1.7-fold, respectively, in Ang II-treated $\mathrm{H} 9 \mathrm{C} 2$ cells. The difference between ANP and BNP regarding the response to Ang II may be associated with systematic errors, including RNA extraction method, PCR cycle number, etc.

mTOR signaling is considered to play a key role in the growth, proliferation, and survival of various cells $(28,29)$. It was previously suggested that RAP can suppress apoptosis in tunicamycin-treated renal proximal tubular cells through inhibition of mTOR (30). Moreover, mTOR signaling can regulate the survival of cardiomyocytes, whereas mTOR inhibitors induce autophagy and inhibit cardiomyocyte death through activating the adenosine monophosphate-activated protein kinase (AMPK) pathway and inhibiting mTOR signaling (31). Intriguingly, mTOR signaling also plays a potential role in cardiac hypertrophy and fibrosis progression (32). Our findings revealed that both ALS and RAP played similar roles in Ang II-induced cardiac hypertrophy by neutralizing p-mTOR expression. Thus, inactivation of mTOR is an important protective mechanism underlying the involvement of ALS in the pathological process of cardiac hypertrophy. It was previously reported that the activation of RAS stimulates the mTOR pathway in HIV-associated nephropathy (33). In addition, ALS and valsartan were found to improve metabolic signaling, oxidative stress, and myocardial tissue remodeling, at least partially, through the activation of p-mTOR expression (24). These results, together with our findings, suggest that ALS, as a RAS inhibitor, may exert RAP-like effects on ISO- and Ang II-induced cardiac hypertrophy.

Regarding the mechanisms underlying the protective role of ALS in the prevention of ISO- and Ang II-induced cardiac hypertrophy, the other possible explanation is that ALS may inhibit the apoptosis of cardiomyocytes. Delaying cardiomyocyte loss may be a promising therapeutic option after injury, as cardiomyocytes are terminally differentiated and have little potential for mitosis (34). 

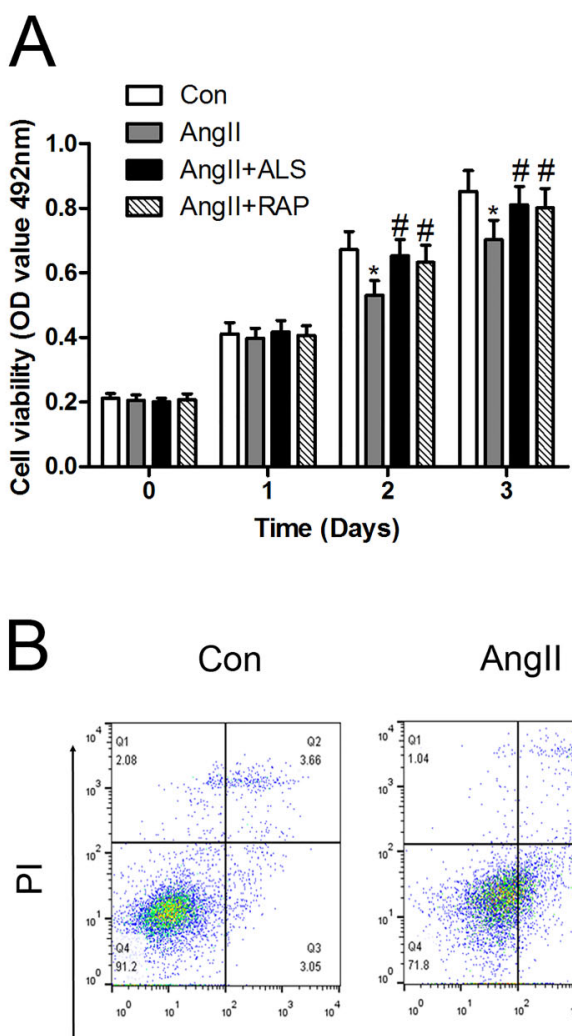

Angll
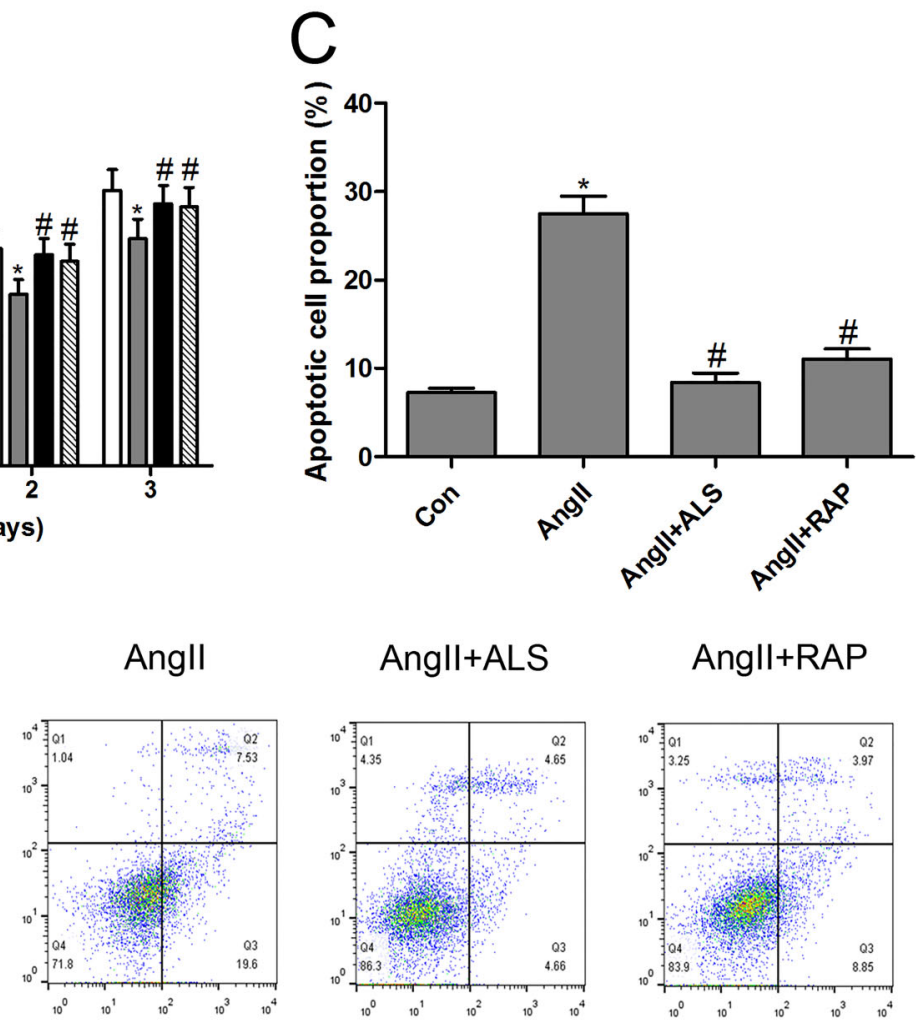

Angll+ALS

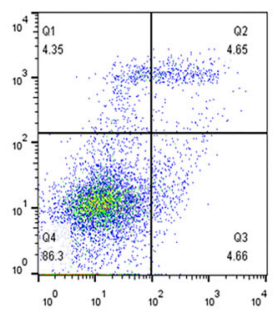

\section{Angll+RAP}

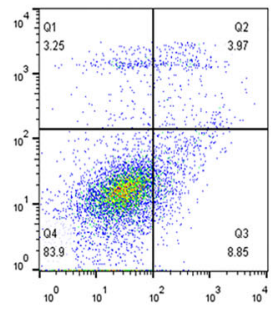

\section{Annexin V-FITC}

Figure 6. Aliskiren (ALS) improved cell viability and inhibited apoptosis in Ang II-treated H9C2 cardiomyocytes. After H9C2 cardiomyocytes were treated with Ang II $(10 \mu \mathrm{M})$, Ang II $(10 \mu \mathrm{M})+\mathrm{ALS}(20 \mu \mathrm{M})$, or Ang II $(10 \mu \mathrm{M})+\operatorname{RAP}(100 \mathrm{nM})$, (A) the cell viability was monitored using MTS assay at 24,48 , and $72 \mathrm{~h}$, and (B and C) apoptosis was analyzed using flow cytometry at $48 \mathrm{~h}(\mathrm{n}=3 \mathrm{per}$ group). Data are reported as means \pm SE. ${ }^{*} \mathrm{P}<0.05$ compared with control; ${ }^{\#} \mathrm{P}<0.05$ compared with the Ang II-treated group (ANOVA followed by Tukey's post hoc test). Ang: angiotensin; RAP: rapamycin.

Westermann et al. (35) demonstrated that the number of apoptotic cardiomyocytes was reduced by ALS after myocardial infarction. Zhang et al. (36) also reported that ALS attenuates myocardial cell apoptosis in aged spontaneously hypertensive rats, accompanied by upregulation of $\mathrm{Bcl}-2$ and survivin and downregulation of Bax and caspase-3. Moreover, a recent study highlighted that ALS alleviates cardiac injury and decreases the levels of apoptosis caused by oxygen-glucose deprivation in mice (34). The present study demonstrated that ALS increased $\mathrm{H} 9 \mathrm{C} 2$ cardiomyocyte viability in vitro under Ang II treatment. Our findings also revealed that ALS significantly attenuated Bax and caspase-3 expression and increased $\mathrm{Bcl}-2$ expression in vivo and in vitro. These findings further support that ALS played a protective role against ISO- and Ang II-induced H9C2 cardiomyocyte injury.

We need to make clear the limitations in our study. First, mTOR signaling, as the direct regulator of autophagy, has been implicated in ISO- or Ang II-induced pathological cardiac hypertrophy $(7,37)$. Autophagy is usually activated and attenuates LV remodeling and cardiomyocyte apoptosis under pathological conditions, accompanied by activation of AMPK and inhibition of mTOR signaling (38), while the effects of ALS on autophagy, as a cascade signaling of mTOR, in the process of ISO- and angiotensin II-induced cardiac hypertrophy, were not explored. In addition, the antifibrotic role of ALS and its underlying mechanism have not been fully elucidated. Furthermore, the effect of ALS on ISO-induced cardiac hypertrophy in vitro remains unclear.

The novel findings presented herein were that ALS attenuated ISO- and Ang II-induced cardiac hypertrophy in vivo and in vitro. It was also demonstrated that ALS treatment suppressed the mTOR and apoptosis pathways in the pathological process of cardiac hypertrophy. These results indicated a novel and direct mechanism by which ALS may be employed in the prevention and treatment of cardiac hypertrophy. 
A
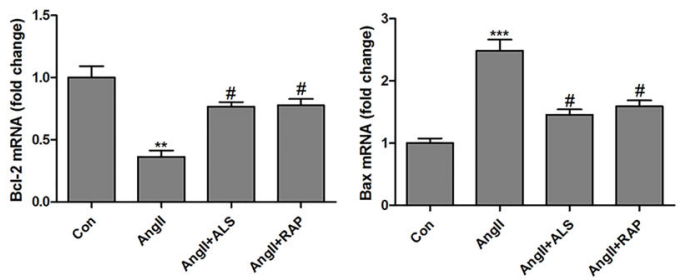

C

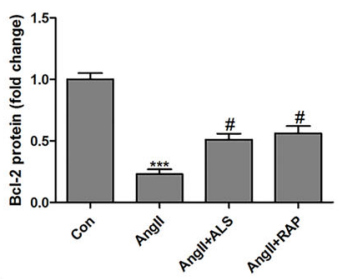

$\mathrm{D}$
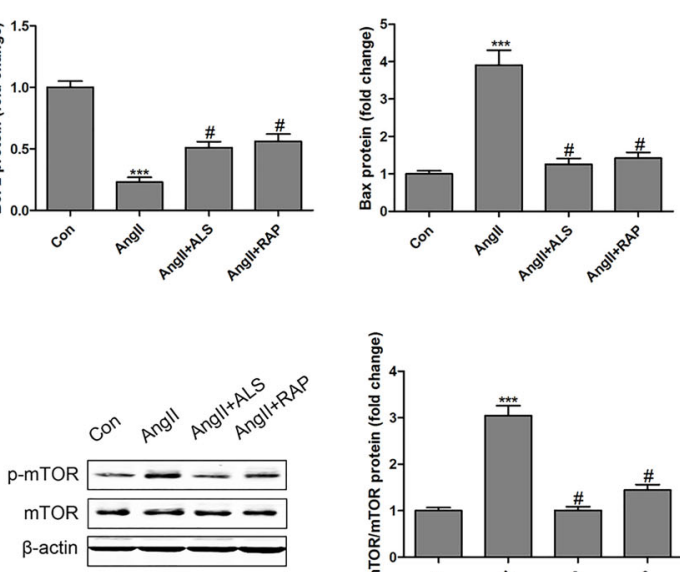

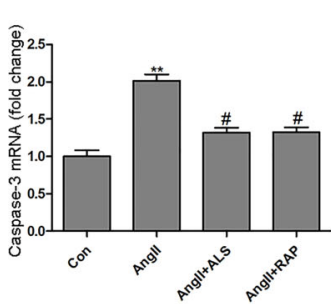

B
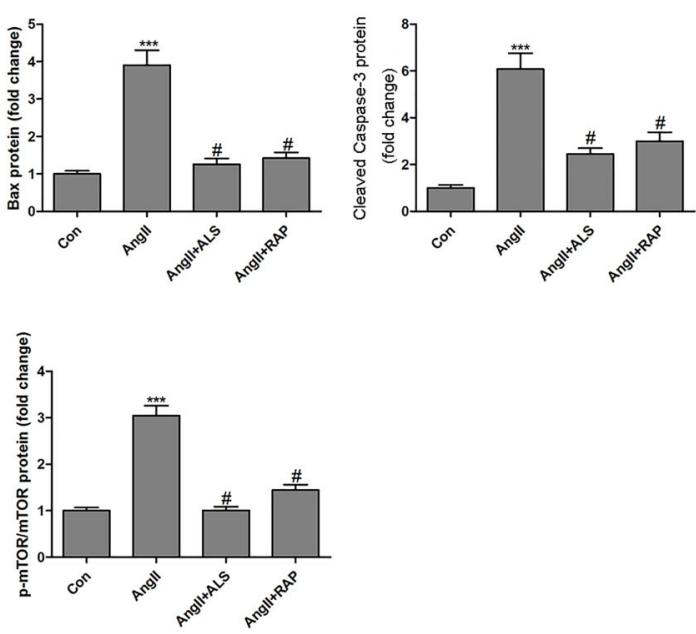

Cleaved Caspase-3
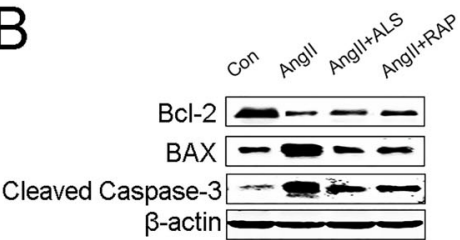

Figure 7. Aliskiren (ALS) inhibited the mTOR and apoptosis pathways in Ang II-treated H9C2 cardiomyocytes. The mRNA expression of Bcl-2, Bax, and caspase-3 was measured by RT-qPCR in H9C2 cardiomyocytes after treatment with Ang II (10 $\mu \mathrm{M})$, Ang II (10 $\mu \mathrm{M})+$ ALS $(20 \mu \mathrm{M})$, or Ang II $(10 \mu \mathrm{M})+$ RAP $(100 \mathrm{nM})$ for $48 \mathrm{~h}$ ( $\mathrm{n}=3$ per group) $(\mathbf{A})$. The protein expression of Bcl-2, Bax, and cleaved caspase-3 was measured by western blotting (B and $\mathbf{C}$ ). The protein expression of mTOR and p-mTOR was measured by western blotting in H9C2 cardiomyocytes after treatment with Ang $\|(10 \mu \mathrm{M})$, Ang $\|(10 \mu \mathrm{M})+\mathrm{ALS}(20 \mu \mathrm{M})$ or Ang II $(10 \mu \mathrm{M})+\mathrm{RAP}(100 \mathrm{nM})$ for $48 \mathrm{~h}(\mathrm{D})$, $\mathrm{n}=3$ per group. Data are reported as means $\pm \mathrm{SE}$. ${ }^{*} \mathrm{P}<0.01$, ${ }^{* \star *} \mathrm{P}<0.001$ compared with control; ${ }^{\#} \mathrm{P}<0.05$ compared with the Ang II-treated group (ANOVA followed by Tukey's post hoc test). mTOR: mammalian target of rapamycin; Ang: angiotensin; RAP: rapamycin.

\section{References}

1. Solomon SD, Appelbaum E, Manning WJ, Verma A, Berglund T, Lukashevich V, et al. Effect of the direct Renin inhibitor aliskiren, the Angiotensin receptor blocker losartan, or both on left ventricular mass in patients with hypertension and left ventricular hypertrophy. Circulation 2009; 119: 530537, doi: 10.1161/CIRCULATIONAHA.108.826214.

2. Maulik SK, Kumar S. Oxidative stress and cardiac hypertrophy: a review. Toxicol Mech Methods 2012; 22: 359-366, doi: $10.3109 / 15376516.2012 .666650$.

3. Li Z, Wang J, Yang X. Functions of autophagy in pathological cardiac hypertrophy. Int J Biol Sci 2015; 11: 672-678, doi: 10.7150/ijbs.11883.

4. Shimizu I, Minamino T. Physiological and pathological cardiac hypertrophy. J Mol Cell Cardiol 2016; 97: 245-262, doi: 10.1016/j.yjmcc.2016.06.001.

5. Zhao QD, Viswanadhapalli S, Williams $P$, Shi Q, Tan C, Yi X, et al. NADPH oxidase 4 induces cardiac fibrosis and hypertrophy through activating Akt/mTOR and NFkappaB signaling pathways. Circulation 2015; 131: 643-655, doi: 10.1161/CIRCULATIONAHA.114.011079.
6. Zhao D, Wang W, Wang H, Peng H, Liu X, Guo W, et al. PKD knockdown inhibits pressure overload-induced cardiac hypertrophy by promoting autophagy via AKT/mTOR pathway. Int J Biol Sci 2017; 13: 276-285, doi: 10.7150/ ijbs.17617.

7. Gao L, Guo Y, Liu X, Shang D, Du Y. KLF15 protects against isoproterenol-induced cardiac hypertrophy via regulation of cell death and inhibition of Akt/mTOR signaling. Biochem Biophys Res Commun 2017; 487: 22-27, doi: 10.1016/j. bbrc.2017.03.087.

8. Du M, Huang K, Gao L, Yang L, Wang WS, Wang B, et al. Nardosinone protects $\mathrm{H} 9 \mathrm{c} 2$ cardiac cells from angiotensin IIinduced hypertrophy. J Huazhong Univ Sci Technolog Med Sci 2013; 33: 822-826, doi: 10.1007/s11596-013-1205-9.

9. Song X, Kusakari Y, Xiao CY, Kinsella SD, Rosenberg MA, Scherrer-Crosbie $M$, et al. mTOR attenuates the inflammatory response in cardiomyocytes and prevents cardiac dysfunction in pathological hypertrophy. Am J Physiol Cell Physiol 2010; 299: C1256-C1266, doi: 10.1152/ajpcell. 00338.2010 . 
10. Kemi OJ, Ceci M, Wisloff U, Grimaldi S, Gallo P, Smith GL, et al. Activation or inactivation of cardiac Akt/mTOR signaling diverges physiological from pathological hypertrophy. J Cell Physiol 2008; 214: 316-321, doi: 10.1002/jcp. 21197.

11. Li L, Zhou N, Gong H, Wu J, Lin L, Komuro I, et al. Comparison of angiotensin II type 1-receptor blockers to regress pressure overload-induced cardiac hypertrophy in mice. Hypertens Res 2010; 33: 1289-1297, doi: 10.1038/ hr.2010.182.

12. Hoshikawa E, Matsumura Y, Kubo T, Okawa M, Yamasaki N, Kitaoka $\mathrm{H}$, et al. Effect of left ventricular reverse remodeling on long-term prognosis after therapy with angiotensinconverting enzyme inhibitors or angiotensin II receptor blockers and beta blockers in patients with idiopathic dilated cardiomyopathy. Am J Cardiol 2011; 107: 1065-1070, doi: 10.1016/j.amjcard.2010.11.033.

13. Stanton A, Jensen C, Nussberger J, O'Brien E. Blood pressure lowering in essential hypertension with an oral renin inhibitor, aliskiren. Hypertension 2003; 42: 1137-1143, doi: 10.1161/01.HYP.0000101688.17370.87.

14. Bin-Dayel AF, Abdel Baky NA, Fadda LM, Mohammad RA, Al-Mohanna F. Effect of aliskiren and carvedilol on expression of $\mathrm{Ca}(2+) /$ calmodulin-dependent protein kinase II delta-subunit isoforms in cardiac hypertrophy rat model. Toxicol Mech Methods 2016; 26: 122-131, doi: 10.3109/ 15376516.2015.1128035.

15. Verdecchia P, Calvo C, Mockel V, Keeling L, Satlin A. Safety and efficacy of the oral direct renin inhibitor aliskiren in elderly patients with hypertension. Blood Press 2007; 16: 381-391, doi: 10.1080/08037050701717014.

16. Gao D, Ning N, Niu X, Wei J, Sun P, Hao G. Aliskiren vs. angiotensin receptor blockers in hypertension: meta-analysis of randomized controlled trials. Am J Hypertens 2011; 24: 613-621, doi: 10.1038/ajh.2011.3.

17. Chen Y, Meng L, Shao H, Yu F. Aliskiren vs. other antihypertensive drugs in the treatment of hypertension: a meta-analysis. Hypertens Res 2013; 36: 252-261, doi: 10.1038/hr.2012.185.

18. Weng LQ, Zhang WB, Ye Y, Yin PP, Yuan J, Wang XX, et al. Aliskiren ameliorates pressure overload-induced heart hypertrophy and fibrosis in mice. Acta Pharmacol Sin 2014; 35: 1005-1014, doi: 10.1038/aps.2014.45.

19. Chowdhury D, Tangutur AD, Khatua TN, Saxena P, Banerjee SK, Bhadra MP. A proteomic view of isoproterenol induced cardiac hypertrophy: prohibitin identified as a potential biomarker in rats. J Trans/ Med 2013; 11: 130, doi: 10.1186/1479-5876-11-130.

20. Saleem N, Prasad A, Goswami SK. Apocynin prevents isoproterenol-induced cardiac hypertrophy in rat. $\mathrm{Mol} \mathrm{Cell}$ Biochem 2018; 445: 79-88, doi: 10.1007/s11010-0173253-0.

21. Livak KJ, Schmittgen TD. Analysis of relative gene expression data using real-time quantitative PCR and the 2(-Delta Delta C(T)) Method. Methods 2001; 25: 402-408, doi: 10.1006/meth.2001.1262.

22. Chan GK, Kleinheinz TL, Peterson D, Moffat JG. A simple high-content cell cycle assay reveals frequent discrepancies between cell number and ATP and MTS proliferation assays. PLoS One 2013; 8: e63583, doi: 10.1371/journal.pone. 0063583.
23. Liang HF, Zhang XZ, Liu BG, Jia GT, Li WL. Circular RNA circ-ABCB10 promotes breast cancer proliferation and progression through sponging miR-1271. Am J Cancer Res 2017; 7: 1566-1576.

24. Whaley-Connell A, Habibi J, Rehmer N, Ardhanari S, Hayden MR, Pulakat L, et al. Renin inhibition and AT(1)R blockade improve metabolic signaling, oxidant stress and myocardial tissue remodeling. Metabolism 2013; 62: 861872, doi: 10.1016/j.metabol.2012.12.012.

25. Wu QQ, Zong J, Gao L, Dai J, Yang Z, Xu M, et al. Sulforaphane protects $\mathrm{H} 9 \mathrm{c} 2$ cardiomyocytes from angiotensin II-induced hypertrophy. Herz 2014; 39: 390-396, doi: 10.1007/s00059-013-3849-4.

26. Tse MM, Aboutabl ME, Althurwi HN, Elshenawy $\mathrm{OH}$, Abdelhamid G, El-Kadi AO. Cytochrome P450 epoxygenase metabolite, 14,15-EET, protects against isoproterenol-induced cellular hypertrophy in $\mathrm{H} 9 \mathrm{c} 2$ rat cell line. Vascul Pharmacol 2013; 58: 363-373, doi: 10.1016/j.vph.2013.02.004.

27. Kralova E, Doka G, Pivackova L, Srankova J, Kuracinova K, Janega $P$, et al. I-arginine attenuates cardiac dysfunction, but further down-regulates alpha-myosin heavy chain expression in isoproterenol-induced cardiomyopathy. Basic Clin Pharmacol Toxicol 2015; 117: 251-260, doi: 10.1111/ bcpt. 12405 .

28. Wang Y, Hu Z, Liu Z, Chen R, Peng H, Guo J, et al. MTOR inhibition attenuates DNA damage and apoptosis through autophagy-mediated suppression of CREB1. Autophagy 2013; 9: 2069-2086, doi: 10.4161/auto.26447.

29. Maiese K, Chong ZZ, Shang YC, Wang S. mTOR: on target for novel therapeutic strategies in the nervous system. Trends Mol Med 2013; 19: 51-60, doi: 10.1016/j.molmed. 2012.11.001.

30. Dong G, Liu Y, Zhang L, Huang S, Ding HF and Dong Z. mTOR contributes to ER stress and associated apoptosis in renal tubular cells. Am J Physiol Renal Physiol 2015; 308: F267-F274, doi: 10.1152/ajprenal.00629.2014.

31. Huang L, Dai K, Chen M, Zhou W, Wang X, Chen J, et al. The AMPK Agonist PT1 and mTOR inhibitor 3HOI-BA-01 protect cardiomyocytes after ischemia through induction of autophagy. J Cardiovasc Pharmacol Ther 2016; 21: 70-81, doi: $10.1177 / 1074248415581177$.

32. Liu B, Wu Z, Li Y, Ou C, Huang Z, Zhang J, et al. Puerarin prevents cardiac hypertrophy induced by pressure overload through activation of autophagy. Biochem Biophys Res Commun 2015; 464: 908-915, doi: 10.1016/j.bbrc.2015. 07.065.

33. Rai $\mathrm{P}$, Lederman $\mathrm{R}$, Haque $\mathrm{S}$, Rehman $\mathrm{S}$, Kumar $\mathrm{V}$, Sataranatrajan K, et al. Renin angiotensin system modulates mTOR pathway through AT2R in HIVAN. Exp Mol Pathol 2014; 96: 431-437, doi: 10.1016/j.yexmp.2014. 04.004

34. Chiang MH, Liang CJ, Liu CW, Pan BJ, Chen WP, Yang YF, et al. Aliskiren improves ischemia- and oxygen glucose deprivation-induced cardiac injury through activation of autophagy and AMP-activated protein kinase. Front Pharmacol 2017; 8: 819, doi: 10.3389/fphar.2017.00819.

35. Westermann D, Riad A, Lettau O, Roks A, Savvatis K, Becher PM, et al. Renin inhibition improves cardiac function and remodeling after myocardial infarction independent of blood pressure. Hypertension 2008; 52: 1068-1075, doi: 10.1161/HYPERTENSIONAHA.108.116350. 
36. Zhang W, Zhao G, Hu X, Wang M, Li H, Ye Y, et al. Aliskirenattenuated myocardium apoptosis via regulation of autophagy and connexin-43 in aged spontaneously hypertensive rats. J Cell Mol Med 2014; 18: 1247-1256, doi: 10.1111/ jcmm.12273.

37. Liu L, Wang C, Sun D, Jiang S, Li H, Zhang W, et al. Calhex 231 ameliorates cardiac hypertrophy by inhibiting cellular autophagy in vivo and in vitro. Cell Physiol Biochem 2015; 36: 1597-1612, doi: 10.1159/000430322.

38. Li MH, Zhang YJ, Yu YH, Yang SH, lqbal J, Mi QY, et al. Berberine improves pressure overload-induced cardiac hypertrophy and dysfunction through enhanced autophagy. Eur J Pharmacol 2014; 728: 67-76, doi: 10.1016/j.ejphar. 2014.01.061. 


\title{
Erratum notice for: "Aliskiren attenuates cardiac dysfunction by modulation of the mTOR and apoptosis pathways" [Braz $\mathrm{J}$ Med Biol Res 2020;53(2): e8793]
}

\author{
Zhengbo Zhao ${ }^{1 * \odot}$, Han $\mathrm{Liu}^{2 * \bullet}$, and Dongmei Guo ${ }^{3 \odot}$ \\ ${ }^{1}$ Department of Cardiovascular Medicine, Jiulongpo District People's Hospital, Chongqing, China \\ ${ }^{2}$ Department of Neurology, Jiulongpo District People's Hospital, Chongqing, China \\ ${ }^{3}$ Department of Cardiovascular Medicine, Nanchuan District People's Hospital, Chongqing, China
}

The authors notified the Editors of the Brazilian Journal of Medical and Biological Research that there was an error in Figure $1 \mathrm{~A}$, due to inappropriate image processing. The conclusion of the results remains unchanged.

The correct Figure 1 is:

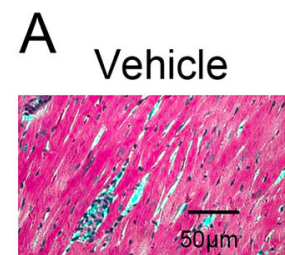

ALS

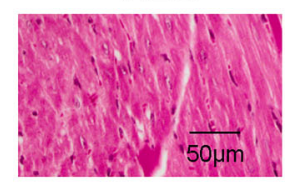

ISO

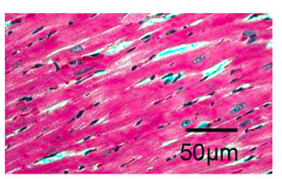

ALS+ISO
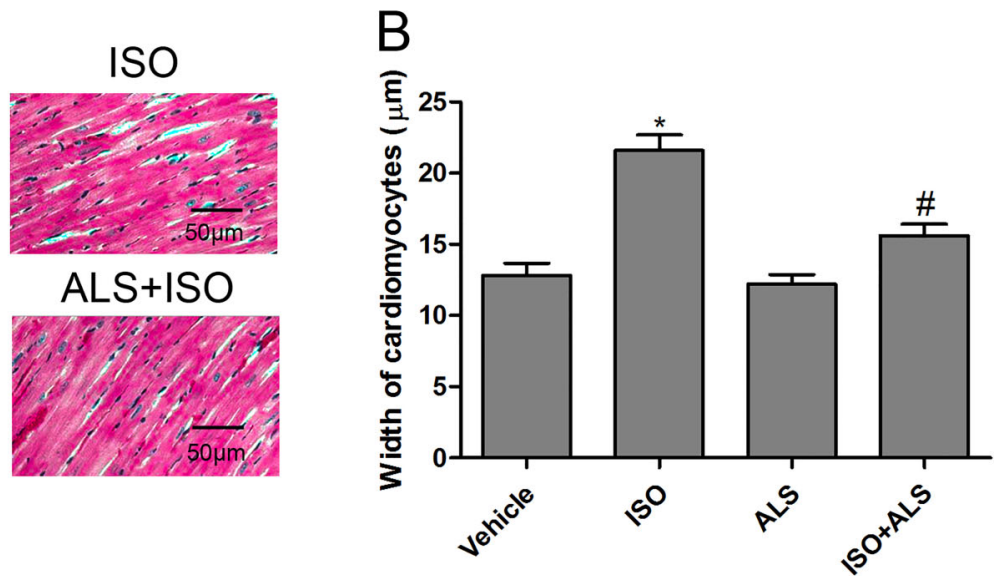

Figure 1. Effect of aliskiren (ALS) on cardiomyocyte size in isoproterenol (ISO)-treated rats. A, Hematoxylin and eosin staining was performed and $(B)$ the width of cardiomyocytes was measured ( $n=6$ per group). Data are reported as means $\pm S E$. ${ }^{*} P<0.05$ compared with vehicle; ${ }^{\mathrm{P}}<0.05$ compared with the ISO-treated group (ANOVA followed by Tukey's post hoc test). 